\title{
Mechanisms for the control of two-mode transient stimulated Raman scattering in liquids
}

\author{
Michael Spanner and Paul Brumer \\ Chemical Physics Theory Group, Department of Chemistry, and Center for Quantum Information and Quantum Control, \\ University of Toronto, Ontario, Toronto, Canada M5S 3 H6 \\ (Received 23 September 2005; published 13 February 2006)
}

\begin{abstract}
Recent adaptive feedback control experiments demonstrated control of transient (i.e. nonimpulsive) Stokes emission from two closely spaced Raman-active modes in liquid methanol [e.g., B. J. Pearson et al., Phys. Rev. A 63, 063412 (2001)]. Optimally shaped pulses were found that selectively excited one of the two Stokes lines alone, optimized emission from both modes together, or completely suppressed all Stokes emission. Here, two general control mechanisms capable of affecting the ratio of intensities of the Stokes lines are identified. The first is operational when the duration of the pump pulse $\left(t_{p}\right)$ is on the order of the collisional dephasing time $\left(t_{d}\right)$. The ratio of the peak heights of the two Stokes lines can then be controlled by simply varying the duration and/or intensity of the pump pulse. The second operates when $1 / t_{p}$ is on the order of the energy separation of the two Raman modes, and hence when the two Raman modes are coupled due to overlapping nonlinear polarizations that drive the stimulated Raman scattering. In this regime, asymmetry in the spectral amplitudes within the pump pulse can control the asymmetry in the peak heights of the Stokes emission. Both these mechanisms have the same clear physical interpretation: shaping the pump pulse controls the nonlinear optical response of the medium, which in turn controls the stimulated Stokes emission, itself a $\chi^{(3)}$ nonlinear effect. In neither mechanism does the ratio of peak heights in the Stokes spectrum reflect directly the ratio of excitedstate populations associated with the two Raman modes, as was assumed in the experiments, nor does the control involve coherent quantum interference effects.
\end{abstract}

DOI: 10.1103/PhysRevA.73.023809

PACS number(s): 42.65.Dr, 42.65.Re, 32.80.Qk

\section{INTRODUCTION}

Adaptive feedback control-loop methods are currently dominating the field of experimental ultrafast laser control of molecules. Although these techniques often enable the experimentalist to find pulse shapes that enhance a particular target, the method offers little understanding of the underlying science, why the optimal pulse shapes work, general rules regarding control efficacy, the role of quantum interference, etc. In this paper we provide the theoretical foundation for a series of adaptive feedback experiments [1-5] that demonstrated control over the Stokes emission from two closely spaced vibrational modes of liquid methanol. Numerous issues regarding these experiments are addressed, and general control mechanisms are identified.

The spontaneous Raman spectrum of liquid methanol shows, among many other features, two Stokes lines with frequency shifts of 2837 and $2947 \mathrm{~cm}^{-1}$ and widths of 17 and $34 \mathrm{~cm}^{-1}$, corresponding to the symmetric and antisymmetric $\mathrm{C}-\mathrm{H}$ stretch modes [6,7]. In the adaptive feedback experiments of Bucksbaum et al. [1-5], a 150 fs laser pulse with peak intensity of $\sim 10^{11} \mathrm{~W} / \mathrm{cm}^{2}$ and central wavelength of $800 \mathrm{~nm}$ was used to drive the stimulated Stokes emission from the two $\mathrm{C}-\mathrm{H}$ modes. The femtosecond pulse bandwidth is on the order of the energy spacing of the two vibrational modes, but is much smaller than the energy separation between the ground vibrational state and the excited vibrational states. This second feature means that the experiments are in the nonimpulsive regime where the molecule has time to vibrate while the pulse is on and where there is no field amplitude near the Stokes frequency in the input laser pulse. Rather, the stimulated Stokes emission is seeded by spontaneous emission and collisional fluctuations [8]. Control was achieved using a genetic algorithm to find the optimal pump pulse shape that selectively stimulates Stokes emission at either one of the two Stokes frequencies. Based on the assumption that the intensity of these Stokes lines directly reflects the populations of the two vibrational modes, Bucksbaum and co-workers [1-5] concluded that selective excitation of either of the two closely space vibrational levels was achieved. In the absence of a firm theoretical footing, these experiments are being touted by many $[1-5,9-13]$ as an example of quantum or coherent control of mode-selective vibrational excitation in the liquid phase.

Indeed, no proper analysis of these experiments has been provided. Most relevant is that a recent proposal for the mechanism of Raman control using a double-Gaussianshaped pump pulse [4] has been shown to be faulty [14]. The present paper and its companion [15] provide a detailed analysis of these experiments, insight into the nature of the optimizing laser pulse, and an understanding of what, in fact, is controlled. Specifically, this paper deals with the control mechanisms present when two-mode stimulated Raman scattering alone is considered, and restricts the analysis to the weakly driven regime where the pump pulse is not strongly modified during the propagation. The mechanisms thus uncovered explain the control reported in the adaptive feedback experiments $[1-3,5]$. The following companion paper, on the other hand, considers the specific control experiment [4] where the double-Gaussian pump pulse was used. In this case, the two-mode stimulated Raman scattering model could only reproduce the experimentally observed control when the pump underwent significant nonlinear modulation. However, in this regime additional nonlinear optical propagation effects, such as self- and cross-phase modulation, become important and can affect the control. The focus of the 
following paper is then on control mechanisms that arise in the strongly driven regime needed to explain the results of Ref. [4]. Together, our two papers give a complete account of the control mechanisms present in both the weakly and strongly driven regimes of two-mode stimulated Raman scattering.

In Sec. II, the theory of stimulated Raman scattering from a single Raman-active transition [8,16-18] is extended to include coupling between two closely spaced modes. The derivation closely follows the standard calculation of stimulated Raman scattering from a single Raman-active mode [8], with the essential difference being the presence of a second mode detuned by a small amount from the first.

Using this model, two distinct mechanisms for the control of the relative Stokes peak heights are found. The first is already present in the theory of stimulated Raman scattering from a single Raman-active transition [16-18] and appears once the pump pulse bandwidth surpasses the collisional line width $\Gamma$ of the Raman mode. The second mechanism arises once the bandwidth of the pump pulse exceeds the energy spacing of the Raman modes resulting in the coupling of multiple Raman modes through the nonlinear polarization $\chi^{(3)}$. Specifically, a qualitative subdivision of control of Stokes emission behavior can be introduced based upon three time scales: the pump pulse duration $t_{p}$, the dephasing time (inverse linewidth) of the Raman transition $t_{d}=1 / \Gamma$, and the beat time between the two excited vibrational states $t_{b}$ $=1 / \Delta$ (here $\Delta=2947-2837 \mathrm{~cm}^{-1}=110 \mathrm{~cm}^{-1}$ ). When $t_{p} \gg t_{d}$, the Raman scattering reaches steady state (i.e., the time dependence of the pump and Stokes pulse envelopes can be neglected) and the stimulated Stokes intensity $I_{S}(z)$ grows exponentially as a function of propagation length $z$ :

$$
I_{s}(z) \propto e^{G_{s s} z / 2},
$$

where $I_{L}$ is the intensity of the pump laser and $G_{s s}$ is the steady-state Raman gain factor [18]. The gain factor depends on the two-photon transition dipole moments coupling the ground state to the Raman active level, the linewidth $\Gamma$ of the transition, and the pump laser intensity $I_{0}$. In this regime (i.e., $t_{p} \gg t_{d}$ ) the $2837 \mathrm{~cm}^{-1}$ mode of methanol dominates the stimulated Raman spectrum by orders of magnitude [6] due to a larger gain factor $G_{s s}$ of this mode than of the $2947 \mathrm{~cm}^{-1}$ mode, regardless of the shape of the pump pulse. Control over the magnitude of the Stokes emission associated with the two modes is, therefore, not possible in this steady-state regime, a result shown in the first part of Sec. III.

A mechanism of control in the transient regime, wherein $t_{p} \leqslant t_{d}$, is the main focus of Sec. III. As the pulse duration approaches or becomes smaller than the dephasing time, the Raman gain changes character and becomes smaller than the steady-state gain $[17,19]$. The Raman gain in this regime, called the transient gain $G_{T}$, continues to decrease as $t_{p} / t_{d}$ $\rightarrow 0$. Furthermore, since the two $\mathrm{C}-\mathrm{H}$ vibrational modes of methanol have different linewidths $\Gamma$ and two-photon coupling strengths, and since the Raman process depends nontrivially on these parameters, the falloff of the Raman gain of the two modes shows a different dependence on $t_{p}$ and on the intensity $I_{0}$ of the pump. The relative peak heights of the two Stokes lines can then be controlled by varying simple control parameters such as the pulse duration and/or peak intensity of the pump, as discussed in Sec. III. The dependence of the relative height of the 2837 and $2947 \mathrm{~cm}^{-1}$ Stokes lines of methanol on $t_{p}$ as $t_{p}$ approaches $t_{d}$ has been seen experimentally since 1969 [6] and, although not explicitly identified as such, is manifest in the experimental results of Bucksbaum et al. [3].

As $t_{p}$ becomes smaller, and $1 / t_{p}$ becomes on the order of the energy spacing between the two Raman modes $\Delta$ $=2947-2837 \mathrm{~cm}^{-1}=110 \mathrm{~cm}^{-1}$ (i.e., $t_{p} \leqslant t_{b}$ ), the two modes necessarily couple through the nonlinear polarization $\chi^{(3)}$ of the medium. The control mechanism that emerges under these conditions is considered in Sec. IV. Specifically, the extended set of stimulated Raman equations for two coupled Raman modes is formally integrated and the nonlinear polarization of the medium is constructed. The resulting expression for the nonlinear polarization $\chi^{(3)}$ shows that overlapping nonlinear polarizations lead to a second mechanism of control of the relative peak heights in the Stokes spectrum. In particular, a pump pulse with asymmetric spectral amplitudes across the bandwidth can be used to manipulate the asymmetry in the peak heights of the Stokes spectrum.

Note that both control mechanisms introduced above need only the lowest-order interactions with the vibrational modes. In such cases, the quantum and classical theories of stimulated Raman scattering give identical results, up to a redefinition of the two-photon coupling constants $[16,20]$. In the classical picture, the vibrational modes are treated as classical damped harmonic oscillators. The resultant control is then devoid of any quantum interference effects. Further, note that we find that in all regimes studied, the relative peak heights seen in the Stokes spectrum do not reflect the relative populations of the two vibrational modes. The situation is therefore different from the case of vibrational control using impulsive Raman excitation [21-23], where control over the vibrational populations was unambiguously demonstrated.

\section{MODEL}

\section{A. Raman scattering}

In Raman scattering, a photon of one frequency $\left(\omega_{L}\right)$ is scattered into a photon of a different frequency $\left(\omega_{S}\right)$, with a concomitant gain or loss of energy in the scattering medium to conserve energy. To construct the appropriate coupled Maxwell and Schrödinger equations for the problem, the electric fields are first written as

$$
\begin{gathered}
\boldsymbol{E}_{T}(\vec{r}, t)=\boldsymbol{E}_{L}(\vec{r}, t)+\boldsymbol{E}_{S}(\vec{r}, t), \\
\boldsymbol{E}_{L}(\vec{r}, t)=E_{L}(\vec{r}, t) e^{-i \omega_{L} t+\overrightarrow{k_{L}} \cdot \vec{r}}+\mathrm{c} \cdot \mathrm{c} ., \\
\boldsymbol{E}_{S}(\vec{r}, t)=E_{S}(\vec{r}, t) e^{-i \omega_{S} t+\vec{k}_{S} \cdot \vec{r}}+\text { c . c . },
\end{gathered}
$$

where $\boldsymbol{E}_{T}$ is the total field, $\boldsymbol{E}_{L}$ is the laser (pump) field, $\boldsymbol{E}_{S}$ is the Stokes field, and c.c. denotes the complex conjugate. In this representation, the Stokes frequency $\omega_{S}$ is the average of the Stokes emission coming from the two Raman modes, a form useful when the two Stokes lines of interest lie within 


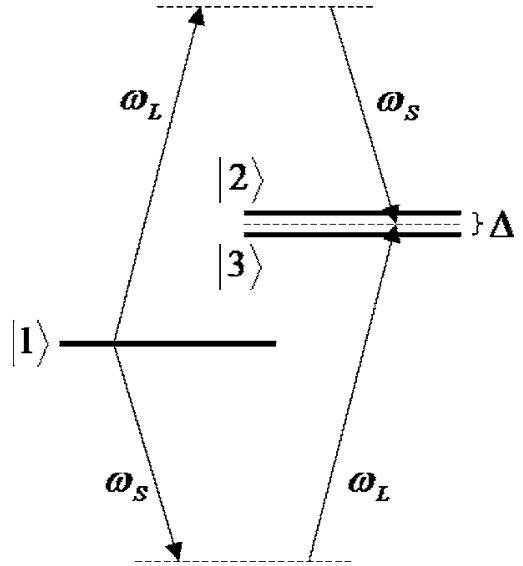

FIG. 1. The pump laser field at frequency $\omega_{L}$ drives Raman emission near frequency $\omega_{S}$ in a three-level system through twophoton Raman couplings. The two excited states $|2\rangle$ and $|3\rangle$ are closely spaced with a small energy separation $\Delta$ that is on the order of the bandwidth of the laser and Stokes pulses.

the finite bandwidth of the Stokes pulse. The pump and Stokes fields follow Maxwell's equation $(i=L, S)$

$$
\left(\nabla^{2}-\frac{1}{c^{2}} \frac{\partial^{2}}{\partial t^{2}}\right) \boldsymbol{E}_{i}(\vec{r}, t)=\frac{4 \pi}{c^{2}} \frac{\partial^{2}}{\partial t^{2}} \boldsymbol{P}_{i}(\vec{r}, t)
$$

where $\boldsymbol{P}_{i}(\vec{r}, t)$ are the polarizations inside the medium.

Within the molecular medium, each molecule is considered as a three-level system, shown in Fig. 1, where the coupling between the ground state $|1\rangle$ and either excited state $|2\rangle,|3\rangle$ occurs through two-photon (i.e., Raman) transitions. The frequencies of the fields and the three-level system are two-photon resonant and related by

$$
\begin{gathered}
\omega_{L}-\omega_{S}=\omega_{31}+\Delta / 2, \\
\omega_{L}-\omega_{S}=\omega_{21}-\Delta / 2, \\
\omega_{21}-\omega_{31}=\Delta,
\end{gathered}
$$

where $\omega_{i j}=\omega_{i}-\omega_{j}, \omega_{i}=\mathcal{E}_{i}, \mathcal{E}_{i}$ is the energy of the $i$ th vibrational state $|i\rangle$, and $\Delta$ is the energy separation of the excited states. (Note that all equations are written in atomic units where $\hbar=e=m_{e}=1$.) The molecular wave function is then written in the basis of molecular eigenstates $|k\rangle$ as

$$
|\Psi(\vec{r}, t)\rangle=\sum_{k} a_{k}(\vec{r}, t)|k\rangle
$$

where $a_{k}(\vec{r}, t)$ are the coefficients at time $t$ and position $\vec{r}$ within the medium. In this basis, the Schrödinger equation for the coefficients becomes

$$
i \frac{\partial}{\partial t} a_{k}(\vec{r}, t)=\sum_{j} H_{k j} a_{j}(\vec{r}, t)
$$

Here, $H_{k j}$ are the matrix elements of the total Hamiltonian

$$
\widehat{H}=\widehat{H}_{0}-\widehat{\boldsymbol{\mu}} \cdot \boldsymbol{E}_{T}(\vec{r}, t),
$$

where $\widehat{H}_{0}$ is the field-free Hamiltonian and $\widehat{\mu}$ is the electric dipole moment operator. We assume that the dipole transitions between the three levels of interest are negligible. That is, levels $|1\rangle,|2\rangle$, and $|3\rangle$ can only interact via Raman transitions through high-lying off-resonant $|m\rangle$ states.

When solving the coupled Maxwell and Schrödinger equations, we assume that the medium is excited in a pencilshaped region of cross-sectional area $A$, leading to onedimensional propagation [8], and that there is negligible depletion of the ground state, i.e., $\left|a_{1}(z, t)\right| \approx 1$. The equations of motion coupling the fields and the density matrix elements are then

$$
\begin{aligned}
\frac{\partial}{\partial z} E_{L}(z, t)= & i \eta_{L} E_{S}(z, t)\left[\kappa_{21} Q_{21}(z, t) e^{-i(\Delta / 2) t}\right. \\
& \left.+\kappa_{31} Q_{31}(z, t) e^{i(\Delta / 2) t}\right]
\end{aligned}
$$

$$
\begin{aligned}
\frac{\partial}{\partial z} E_{S}(z, t)= & i \eta_{S} E_{L}(z, t)\left[\kappa_{21} Q_{21}^{*}(z, t) e^{i(\Delta / 2) t}\right. \\
& \left.+\kappa_{31} Q_{31}^{*}(z, t) e^{-i(\Delta / 2) t}\right],
\end{aligned}
$$

$$
\frac{\partial}{\partial t} Q_{21}(z, t)+\Gamma_{2} Q_{21}(z, t)=i \kappa_{21} E_{L}(z, t) E_{S}^{*}(z, t) e^{i(\Delta / 2) t}+\mathcal{F}_{2},
$$

$$
\frac{\partial}{\partial t} Q_{31}(z, t)+\Gamma_{3} Q_{31}(z, t)=i \kappa_{31} E_{L}(z, t) E_{S}^{*}(z, t) e^{-i(\Delta / 2) t}+\mathcal{F}_{3},
$$

$$
\begin{aligned}
\frac{\partial}{\partial t} Q_{22}(z, t)+\gamma_{2} Q_{22}(z, t)= & -i \kappa_{21} E_{L}^{*}(z, t) E_{S}(z, t) e^{-i(\Delta / 2) t} Q_{21}(z, t) \\
& + \text { c. . . . }
\end{aligned}
$$

$$
\begin{aligned}
\frac{\partial}{\partial t} Q_{33}(z, t)+\gamma_{3} Q_{33}(z, t)= & -i \kappa_{31} E_{L}^{*}(z, t) E_{S}(z, t) e^{i(\Delta / 2) t} Q_{31}(z, t) \\
& + \text { c. . . . }
\end{aligned}
$$

where $Q_{n m}(z, t)=\rho_{n m}(z, t) e^{-i \omega_{n m} t}$ are the slowly varying envelopes of the density matrix elements, $\gamma_{i}$ is the decay rate of the excited-state populations, $\Gamma_{i}$ is the collision linewidth of the $i$ th Raman transitions that accounts for dephasing of the vibrational response, and the $\mathcal{F}_{i}$ represent random Langevin forces due to collisions in the liquid [8]. The constants $\kappa_{i 1}$ and $\eta_{n}$ are

$$
\kappa_{i 1}=\sum_{m} \mu_{i m} \mu_{1 m}\left(\frac{1}{\omega_{m 1}-\omega_{L}}+\frac{1}{\omega_{m 1}+\omega_{S}}\right)
$$

and 


$$
\eta_{n}=\frac{2 \pi N \omega_{n}}{c} \quad(n=L, S),
$$

where the $\mu_{i j}$ are the transition dipole matrix elements connecting the ground state $|1\rangle$ and Raman-active levels $|2\rangle$ and $|3\rangle$ to intermediate $|m\rangle$ states, and $N$ is the number density of molecules per unit volume.

Equations (8) basically represent the Raman scattering of the laser field into the Stokes field from two uncoupled Raman-active modes of a single molecule. Identical equations would result from a mixture of molecules, each with only one of the two modes. Two remarks are of note. The resultant equations are consistent with a straightforward extension of known equations for the Raman scattering from a single level [8,16-18] with the appropriate detunings. Second, a classical treatment [16] of the molecules, where the medium is envisioned to be composed of a mixture of two types of classical damped harmonic oscillators (one with an oscillation frequency of $\omega_{31}$ and the other with frequency $\omega_{21}$ ), would give the same equations of motion as Eqs. (8a)-(8d), up to a redefinition of the coupling constants $\kappa_{21}$ and $\kappa_{31}$. In the classical model [16], the molecular fields $Q_{21}(z, t)$ and $Q_{31}(z, t)$ represent the amplitude of oscillation along the normal coordinate associated with the Raman modes at the frequencies $\omega_{21}$ and $\omega_{31}$.

\section{B. Modeling the experiments}

In the experiments of Bucksbaum et al. [1-5], no Stokes field is initially present. The initial condition at $z=0$ then consists of a specified laser pulse $E_{L}(z=0, t)=E_{L 0}(t)$ and an empty Stokes wave $E_{S}(z=0, t)=0$. The molecular vibrations are seeded by spontaneous emission and/or collisional fluctuations. In our model, spontaneous emission is modeled semiclassically by imposing small amplitude stochastic initial conditions on $Q[8,24]$, while the collisional fluctuations are included through the stochastic Langevin forces $\mathcal{F}_{i}$ [8]. Computationally, the spontaneous emission and random collisional effects obey Gaussian random statistics:

$$
\left\langle Q_{i 1}^{*}(z, t=0) Q_{i 1}\left(z^{\prime}, t=0\right)\right\rangle=(1 / \rho) \delta\left(z-z^{\prime}\right)
$$

and

$$
\left\langle F_{i}^{*}(z, t) F_{i}\left(z^{\prime}, t^{\prime}\right)\right\rangle=\left(2 \Gamma_{i} / \rho\right) \delta\left(z-z^{\prime}\right) \delta\left(t-t^{\prime}\right)
$$

where $\rho=N A$ is the number density of molecules per unit length along the propagation direction. The results of calculations are averaged over these initial fluctuations $[8,24]$.

In the calculations that follow, no attempt is made to quantitatively reproduce the experimental results; instead we focus on obtaining the observed control qualitatively. There are a number of reasons for this approach. First, our analysis reveals that there are many nonlinear optical processes involved in the experimental setup and that the calculated Stokes spectrum is very sensitive to the particular balance of all effects present [15]. Second, the Stokes spectrum of methanol near the two lines of interest is complicated by the presence of weaker lines at 2885, 2918, and $2987 \mathrm{~cm}^{-1}$ [7]. Although these lines are of considerably lower intensity, they may become more important in the stimulated regime once the dominant lines are suppressed due to the shaping of the pump pulse. Hence, a careful treatment of the line shapes and two-photon coupling parameters of all five lines would likely be necessary to properly reproduce the control results. Finally, pulse shaping can often disturb the spatial profile of the input pulse, resulting in nonuniform phase fronts from pulse shape to pulse shape. Such varying spatial profile will affect the nonlinear propagation, most notably the selffocusing effects, which will in turn affect the Stokes emission. The spatial profile of the pulse shapes was not characterized in the experiment, and hence one cannot expect to quantitatively reproduce the measured Stokes spectra without far more experimental information.

The experiments were conducted using a titanium:sapphire laser $(800 \mathrm{~nm})$ to generate a pump pulse. The central Stokes wavelength, deduced by subtracting the average Raman shift from the pump wavelength, is $1025 \mathrm{~nm}$. The initial Ti:sapphire pulses have a bandwidth corresponding to $\sim 150 \mathrm{fs}$, while after pulse shaping the pump pulse varied in duration from 0.15 to $5 \mathrm{ps}$. The pump pulse was focused into a $10 \mathrm{~cm}$ cell containing liquid methanol using a $f=40 \mathrm{~cm}$ focal length lens and the peak intensity in the cell was estimated to be $I_{0} \sim 10^{11} \mathrm{~W} / \mathrm{cm}^{2}$. Assuming a typical beam diameter before the focusing lens of $D=0.5 \mathrm{~cm}$ and a diffraction-limited (i.e., ideal) focus diameter $d=0.44 f \lambda / D$, Gaussian beam formulas give an ideal Rayleigh range (the focus width along the propagation direction) of about $0.15 \mathrm{~cm}$. Below, we restrict our calculations to propagation lengths on this order while setting the cross-sectional area of the pencil-shaped volume used in the calculations to be $A$ $=\pi d^{2}$. The peak input intensity of the pump laser is kept near $\sim 10^{11} \mathrm{~W} / \mathrm{cm}^{2}$ and the duration $\sim 1 \mathrm{ps}$. The two-photon coupling parameters are freely tuned to control the regime of stimulated Raman emission (i.e., below or above saturation of the pump pulse) while the ratio of the coupling parameter for the two modes is set to mimic the experimental Stokes spectrum observed with an unshaped pump pulse. The line widths are $\Gamma_{2}=17 \mathrm{~cm}^{-1}$ for the $2837 \mathrm{~cm}^{-1}$ mode and $\Gamma_{3}$ $=34 \mathrm{~cm}^{-1}$ for the $2847 \mathrm{~cm}^{-1}$ mode. The decay rates $\gamma_{i}$ are assumed to be negligible in the numerical simulations.

A simple order-of-magnitude estimate for the molecular constants $\kappa_{i 1}$ can be made by relating them to the linear index of refraction [20]

$$
n^{2}=1+4 \pi N \chi^{(1)}
$$

where

$$
\chi^{(1)}=\sum_{m}\left|\mu_{1 m}\right|^{2}\left(\frac{1}{\omega_{m 1}-\omega}+\frac{1}{\omega_{m 1}+\omega}\right) .
$$

The $\kappa_{i 1}$ are calculated using a sum over dipole moments, Eq. (9), very similar to the sum defining $\chi^{(1)}$. However, the $\kappa_{i j}$ depend on the sum of products of different dipole matrix elements, as opposed to the product of squares of the same matrix elements used in Eq. (14). Since the products can be both positive and negative, whereas the squares are always positive, we expect the $\kappa_{i j}$ to be roughly of the same order of magnitude as $\chi^{(1)}$ but a bit smaller. We then restrict the values of $\kappa_{i j}$ to be near the rough estimate of $\chi^{(1) / 10}$ 
$\approx 2.78$ a.u., where $\chi^{(1)}$ was evaluated from Eq. (13) using $n=1.329$ and $N=0.7917 \mathrm{~g} / \mathrm{cm}^{3}$ corresponding to liquid methanol [25].

\section{UNCOUPLED REGIME}

Consider first the case of uncoupled Raman levels in the regime where the pump depletion is negligible. From Eqs. (8), the uncoupled equations of motion for this scenario can be written as

$$
\begin{gathered}
\frac{\partial E_{S}^{(i)}}{\partial z}=i \eta_{S} \kappa_{21} Q_{i 1}^{*} E_{L}, \\
\frac{\partial Q_{i 1}}{\partial t}+\Gamma_{i} Q_{i 1}=i \kappa_{i 1} E_{L} E_{S}^{(i)^{*}}, \\
\frac{\partial Q_{i i}}{\partial t}+\gamma_{i} Q_{i i}=-i \kappa_{i 1} E_{L}^{*} E_{S}^{(i)} Q_{i 1}+\text { c. c . },
\end{gathered}
$$

where $i=2,3$. Here, it proves convenient to use a detuning for each mode $|i\rangle$ and Eqs. (15) use two different Stokes fields $E_{S}^{(i)}$ that interact independently with the corresponding mode. Equations (15a) and (15b) are the standard equations describing stimulated Raman scattering from a single mode [8,16-18], extended to two uncoupled modes.

\section{A. Steady-state regime}

The steady-state solution discussed in the Introduction can be readily found by neglecting the time derivatives and assuming that the pump laser amplitude is space-time independent [18]. Applying these assumptions to Eqs. (15) leads to the equation

$$
\frac{\partial E_{S}^{(i)}}{\partial z}=\left(\eta_{S} \kappa_{i 1}^{2}\left|E_{L}\right|^{2} / \Gamma_{i}\right) E_{S}^{(i)}
$$

which can be solved to give

$$
E_{S}^{(i)}(z)=E_{S 0}^{(i)} \exp \left(G_{s s}^{(i)} z / 2\right)
$$

where $G_{s s}^{(i)}=2 \eta_{S}\left|\kappa_{i 1}\right|^{2}\left|E_{L}\right|^{2} / \Gamma_{i}$ is the steady-state Raman gain factor and $E_{S 0}^{(i)}$ is the initial Stokes field. Although no initial Stokes field is technically present, the fluctuations of $Q_{i 1}$ that model spontaneous emission and collisions create a small fluctuating amplitude in the Stokes field. For the present discussion, these random fluctuations can be thought of as the initial state $E_{S 0}^{(i)}$ of the Stokes field that subsequently undergoes exponential growth according to Eq. (17). The vibrational excitation follows a similar exponential growth given by

$$
\begin{gathered}
Q_{i 1}(z)=i\left(\frac{\kappa_{i 1}}{\Gamma_{i}}\right) E_{L} E_{S 0}^{(i) *} \exp \left(G_{s s}^{(i)} z / 2\right), \\
Q_{i i}(z)=2\left(\frac{\left|\kappa_{i 1}\right|^{2}}{\Gamma_{i} \gamma_{i}}\right)\left|E_{L}\right|^{2}\left|E_{S 0}^{(i)}\right|^{2} \exp \left(G_{s s}^{(i)} z\right) .
\end{gathered}
$$

This solution is valid when the pump pulse is much longer than the characteristic dephasing time $t_{d}^{(i)}=1 / 2 \Gamma_{i}$ of the Raman transitions.
The control experiments on methanol were not performed in the steady-state regime. Nonetheless, examining the steady-state solution is instructive for two reasons. First, it demonstrates that control over the ratio of two particular peak heights in the emitted Stokes spectrum, or over the vibrational excitations, is not possible in this regime-the mode with the larger $\left|\kappa_{i 1}\right|^{2} / \Gamma_{i}$, and hence larger gain factor $G_{s s}^{(i)}$, will always dominate the Stokes emission. Second, it demonstrates that, even in the simplest case of stimulated Raman scattering, the ratio of two peak heights seen in the Stokes spectrum does not necessarily reflect the ratio of excited-state populations of the associated Raman modes, a result of the factor $\left|\kappa_{i 1}\right|^{2} / \Gamma_{i} \gamma_{i}$ in Eq. (18a). For example, even if the ratio of Stokes intensities is unity for two particular emission lines (i.e., if the Raman gains are equal implying that $\left.\left|\kappa_{21}\right|^{2} / \Gamma_{2}=\left|\kappa_{31}\right|^{2} / \Gamma_{3}\right)$, the ratio of the related vibrational populations would be $\left(\left|\kappa_{21}\right|^{2} / \Gamma_{2} \gamma_{2}\right) /\left(\left|\kappa_{31}\right|^{2} / \Gamma_{3} \gamma_{3}\right)=\gamma_{3} / \gamma_{2}$, which is not necessarily 1 .

\section{B. Transient regime}

When the pump pulse $t_{p}$ duration approaches the time scale of collisional dephasing $t_{d}^{(i)}=1 / 2 \Gamma_{i}$, the time derivatives in Eqs. (15) cannot be neglected. This is called the transient regime. If, for example, the pump pulse has a square shape, so that the pump amplitude is space-time independent in the laser-molecule interaction region, an approximate solution for exponential growth of the Stokes mode can be found [18] and is given by

$$
E_{S}^{(i)} \sim \exp \left[\left(2 G_{s S}^{(i)} \Gamma_{i} z t_{p}\right)^{1 / 2}-\Gamma_{i} t_{p}\right] .
$$

The Raman gain now depends nontrivially on the pulse duration $t_{p}$. A general dependence on the ratio of the pulse duration to the dephasing time $t_{p} / t_{d}^{(i)}$, mentioned in the Introduction, can be discerned from the second term in the exponent $\Gamma_{i} t_{p}=t_{p} / t_{d}^{(i)}$. However, the first term retains a dependence on the two-photon coupling $\kappa_{i 1}\left(\operatorname{via} G_{s s}^{(i)}\right)$ and the pump pulse intensity (note that the factor $G_{s s}^{(i)} \Gamma_{i}$ is independent of $\Gamma_{i}$ since $\Gamma_{i}$ also appears in the denominator of $G_{s s}^{(i)}$ ). The growth of the Stokes emission thus depends on a sensitive balance of all parameters involved. Note that the molecular parameters $\Gamma_{i}$ and $\kappa_{i 1}$ are of the same order of magnitude for the two Raman modes of methanol of interest. Thus, the relative gain in the two modes can be simply controlled by varying the pulse duration $t_{p}$ near the characteristic and/or the maximum pump intensity $I_{0}$, provided that the parameters $\kappa_{i 1}$ and $\Gamma_{i}$ differ for the two modes.

As an example, Fig. 2 plots the square-pulse transient gain solution Eq. (19) for the two Raman modes to show the dependence on the pump pulse duration $t_{p}$ and intensity $I_{0}$. The two-photon couplings used were $\kappa_{31}=3.1 \mathrm{a}$.u. (2947 $\mathrm{cm}^{-1}$ mode) and $\kappa_{21}=2.5$ a.u. $\left(2837 \mathrm{~cm}^{-1}\right.$ mode) with propagation length $z=0.1 \mathrm{~cm}$, where the $\kappa_{i 1}$ were set to loosely mimic the experimental Stokes spectrum [1-3] (ratio of peak heights and Stokes intensity relative to the pump pulse) driven by the unshaped pump pulse. In panel (a), the pump pulse duration is varied from 0.5 to $1.5 \mathrm{ps}$. For short pulse durations, the $2837 \mathrm{~cm}^{-1}$ mode is seen to dominate. 

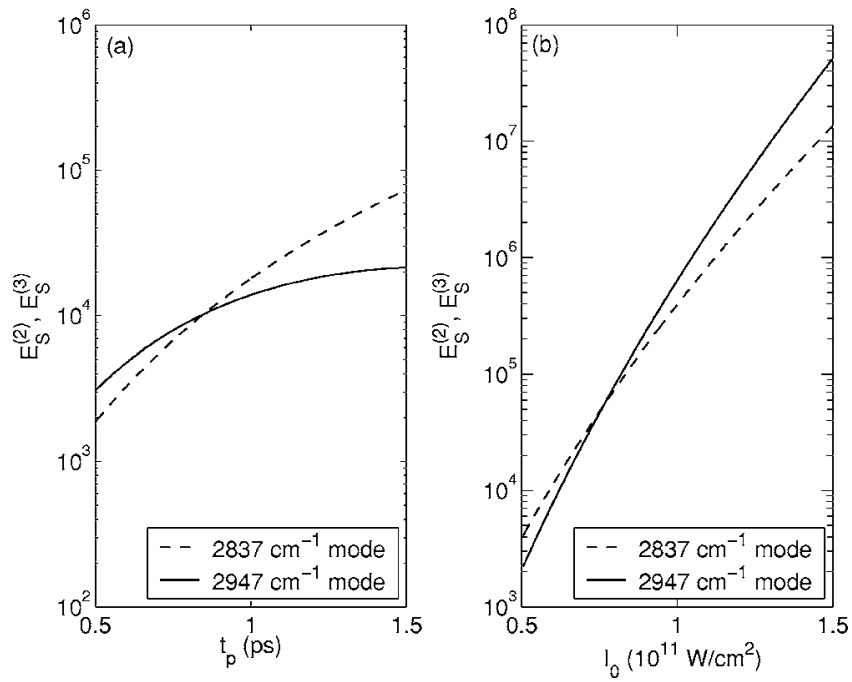

FIG. 2. Dependence of the Stokes fields $E_{S}^{(i)}$ for an incident square pulse in the transient regime on (a) the pulse duration $t_{p}$ and (b) the intensity $I_{0}$ of the pump pulse. The dashed curves correspond to the $2837 \mathrm{~cm}^{-1}$ mode $(|2\rangle)$ and the solid curves correspond to the $2947 \mathrm{~cm}^{-1}$ mode $(|3\rangle)$.

However, as the pulse duration is increased, the $2947 \mathrm{~cm}^{-1}$ mode becomes stronger and starts to dominate. In panel (b), the pump intensity is varied from $0.5 \times 10^{11}$ to 1.5 $\times 10^{11} \mathrm{~W} / \mathrm{cm}^{2}$. Again, there is a switch in the dominant mode as the intensity is varied.

For the case of a more realistic Gaussian pump pulse

$$
E_{L}(z=0, t)=E_{0} \exp \left[-4 \ln 2\left(t / t_{p}\right)^{2}\right]
$$

the two-mode Raman equations (8) are solved numerically. Examples of controlled Stokes emission using this pulse are shown in Figs. 3 and 4, where, once again, $\kappa_{31}=3.1$ a.u. and $\kappa_{21}=2.5 \mathrm{a}$.u. Only the initial fluctuations corresponding to the Langevin force $\mathcal{F}$ were included in these simulationsthe collisional fluctuations of the excited-state populations alone are enough to seed the Raman emission. The left columns of the figures show the calculated Stokes spectrum at the output of the medium, here $z=0.15 \mathrm{~cm}$. In Fig. 3, the pump intensity is set to $I_{0}=10^{11} \mathrm{~W} / \mathrm{cm}^{2}$ while the pump duration is varied: $t_{p}=$ (a) 1.5 , (b) 1 , and (c) 0.5 ps. In Fig. 4, the pump duration is set to $t_{p}=1 \mathrm{ps}$ while the pump intensity is varied: $I_{0}=$ (a) $0.5 \times 10^{11}$, (b) $1 \times 10^{11}$, and (c) 1.5 $\times 10^{11} \mathrm{~W} / \mathrm{cm}^{2}$. The ratio of peak heights seen in the numerical Stokes spectra is seen to depend on the pump pulse duration and intensity in qualitatively the same way as the analytical result modeling the square pump pulse Fig. 2. Thus, by varying simple pulse parameters such as pulse duration and intensity in the transient regime one can control the Stokes peak heights, in agreement with expectations based on the response of a single uncoupled Raman transition discussed above.

To follow the dynamics of the populations, we define the population asymmetry $\mathcal{R}(z, t)$ as
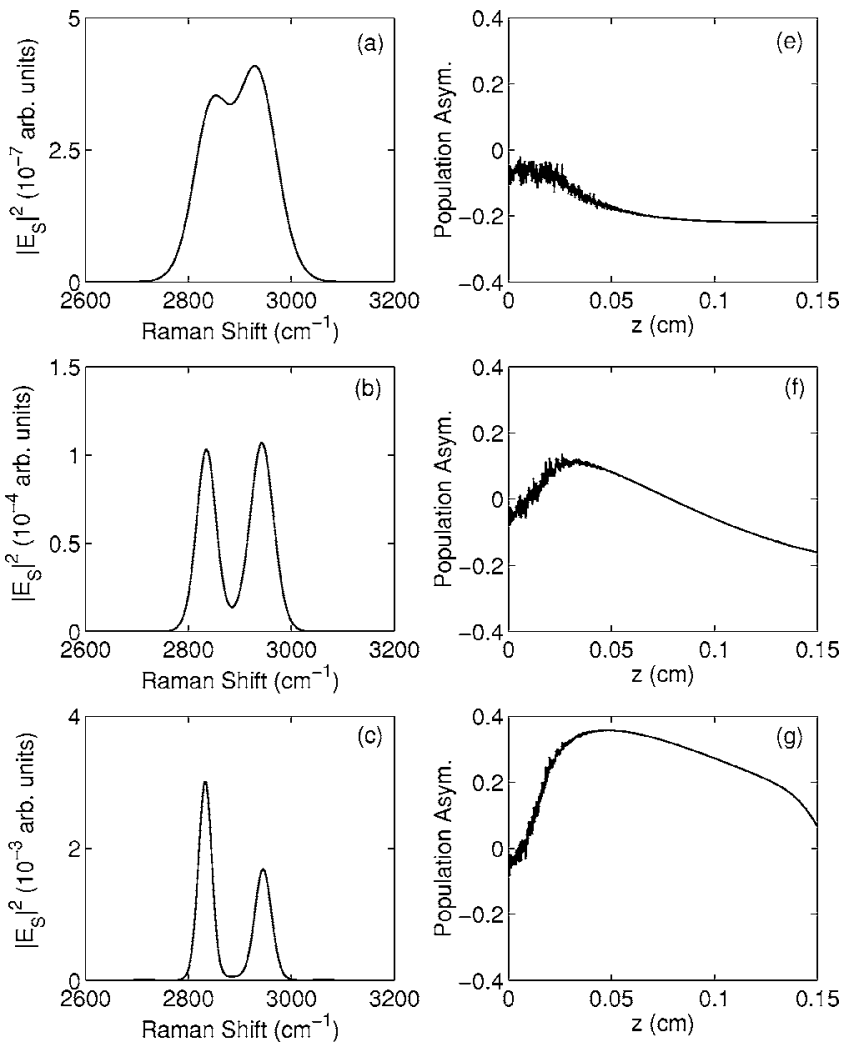

FIG. 3. Control of the Stokes emission by varying the time duration of the pump pulse $t_{p}$. (a), (b), and (c) show the Stokes spectrum for $t_{p}=0.5,1.0$, and $1.5 \mathrm{ps}$, respectively. (e), (f), and (g) to the right of each Stokes spectrum show the corresponding population asymmetry $\mathcal{R}\left(z, t_{f}\right)$, evaluated at the end of the pulse $t=t_{f}$, as a function of propagation length through the medium.

$$
\mathcal{R}(z, t)=\frac{Q_{22}(z, t)-Q_{33}(z, t)}{Q_{22}(z, t)+Q_{33}(z, t)} .
$$

$\mathcal{R}=1$ means that all the excited population is in the $|2\rangle$ mode $(\mathcal{R}=-1$ is all excited population in $|3\rangle$ ) while $\mathcal{R}=0$ indicates equal populations in the two excited modes. To the right of each Stokes spectrum in Figs. 3 and 4 is a plot of the final asymmetry $\mathcal{R}\left(z, t_{f}\right)$ as a function of propagation length through the medium $z$, where $t=t_{f}$ denotes the end of the pump pulse. For all cases, the asymmetry is seen to depend on the propagation length and in most cases even changes sign as the pump propagates along $z$. Note that the asymmetry at the end of the medium $(z=0.15 \mathrm{~cm})$ does not always reflect the asymmetry seen in the peak heights of the corresponding Stokes emission. This is due to both collisional effects acting on the molecular states as well as propagation effects of the Stokes field. Further, we note that, after averaging over collisional fluctuations, the off-diagonal density matrix elements $Q_{i j}$ are zero. The same result appears if averaging over spontaneous emission is included. The control is therefore a completely incoherent effect at the molecular level.

\section{TRANSIENT COUPLED REGIME}

We now consider the transient coupled regime wherein the bandwidth of the pump pulse is greater than the energy 

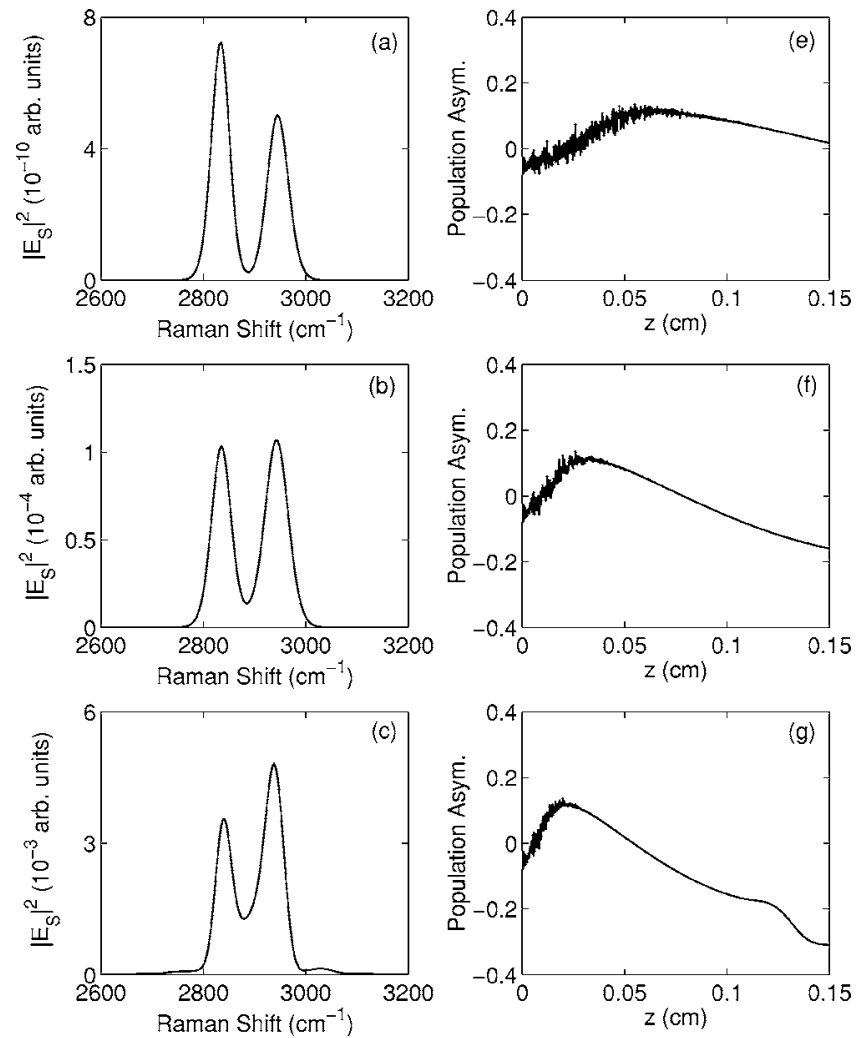

FIG. 4. Control of the Stokes emission by varying the maximum intensity of the pump pulse. (a), (b), and (c) show the Stokes spectrum for $I_{0}=0.5,1.0$, and $1.5 \times 10^{11} \mathrm{~W} / \mathrm{cm}^{2}$, respectively. (e), (f), and $(\mathrm{g})$ to the right of each Stokes spectrum show the corresponding population asymmetry $\mathcal{R}\left(z, t_{f}\right)$, evaluated at the end of the pulse $t$ $=t_{f}$, as a function of propagation length through the medium.

separation $\Delta$. Consider first the formal solution to Eqs. (8c) and (8d) describing the driven component of the off-diagonal density matrix elements:

$$
\begin{aligned}
& Q_{21}(z, t)=i \kappa_{21} e^{-\Gamma_{2} t} \int_{-\infty}^{t} d t^{\prime} e^{\Gamma_{2} t^{\prime}} E_{L}\left(z, t^{\prime}\right) E_{S}^{*}\left(z, t^{\prime}\right) e^{i(\Delta / 2) t^{\prime}} \\
& Q_{31}(z, t)=i \kappa_{31} e^{-\Gamma_{3} t} \int_{-\infty}^{t} d t^{\prime} e^{\Gamma_{3} t^{\prime}} E_{L}\left(z, t^{\prime}\right) E_{S}^{*}\left(z, t^{\prime}\right) e^{-i(\Delta / 2) t^{\prime}}
\end{aligned}
$$

The polarization of the medium at the Stokes frequency is given by [8]

$$
P_{S}(z, t)=N E_{L}(t)\left[\kappa_{21} Q_{21}^{*}(z, t) e^{i(\Delta / 2) t}+\kappa_{31} Q_{31}^{*}(z, t) e^{-i(\Delta / 2) t}\right] .
$$

Using the formal solution for the amplitudes and Fouriertransforming $P_{S}(z, t)$ with respect to $t$ gives the polarization

$$
\begin{aligned}
P_{S}(z, \Omega)= & N\left(\left|\kappa_{21}\right|^{2} \iint d \omega^{\prime} d \omega^{\prime \prime}\right. \\
& \times \frac{E_{L}\left(z, \Omega+\omega^{\prime}-\omega^{\prime \prime}\right) E_{L}^{*}\left(z, \omega^{\prime}\right) E_{S}\left(z, \omega^{\prime \prime}\right)}{\left(\omega^{\prime \prime}-\omega^{\prime}+\Delta / 2+i \Gamma_{2}\right)} \\
& +\left|\kappa_{31}\right|^{2} \iint d \omega^{\prime} d \omega^{\prime \prime} \\
& \left.\times \frac{E_{L}\left(z, \Omega+\omega^{\prime}-\omega^{\prime \prime}\right) E_{L}^{*}\left(z, \omega^{\prime}\right) E_{S}\left(z, \omega^{\prime \prime}\right)}{\left(\omega^{\prime \prime}-\omega^{\prime}-\Delta / 2+i \Gamma_{3}\right)}\right) .
\end{aligned}
$$

The Stokes polarization $P_{S}(z, \Omega)$ contains two contributions that generate interferences in the emitted Stokes radiation at frequency $\Omega$. The first type is the interference within a single Raman transition, due to the presence of a bandwidth. It appears in the polarization as a double integral over $\omega^{\prime}$ and $\omega^{\prime \prime}$ representing all possible combinations of photons that can emit a new photon at frequency $\Omega$. The second type of interference is between the Stokes emission coming from levels $|2\rangle$ and $|3\rangle$, and appears as the sum of the two double integrals.

The denominator in the double integrals weights the integrands heavily near $\omega^{\prime \prime}-\omega^{\prime}=\Delta / 2$ or $\omega^{\prime \prime}-\omega^{\prime}=-\Delta / 2$. For example, for the $\Gamma_{i}=0$ case discussed below, approximating these factors by $\delta$ functions

$$
\frac{1}{\left(\omega^{\prime \prime}-\omega^{\prime} \pm \Delta / 2+i \Gamma_{i}\right)} \sim \delta\left(\omega^{\prime \prime}-\omega^{\prime} \pm \Delta / 2\right)
$$

leads to the following simple approximation to the Stokes polarization:

$$
\begin{aligned}
P_{S}(z, \Omega) \sim & N\left(\left|\kappa_{21}\right|^{2} E_{L}(z, \Omega+\Delta / 2) \int d \omega E_{L}^{*}(z, \omega+\Delta / 2)\right. \\
& \times E_{S}(z, \omega)+\left|\kappa_{31}\right|^{2} E_{L}(z, \Omega-\Delta / 2) \\
& \left.\times \int d \omega E_{L}^{*}(z, \omega-\Delta / 2) E_{S}(z, \omega)\right)
\end{aligned}
$$

As a consequence, the resultant Stokes polarization can then be thought of as being made up of two "images" of the pump spectrum that are shifted by $\Delta$. This form suggests a possible $E_{L}$ pulse shape that could control the relative peak height of the Stokes emission. If the bandwidth of the pump pulse exceeds $\Delta$, and if the pump spectrum has a skewed shape, the emission from the two Raman modes can overlap more favorably at one of the Stokes lines than the other. Owing to the fact that the polarization $P_{S}$ feeds an exponentially growing process as the fields propagate along $z$, this small asymmetry in the instantaneous emission of the Stokes fields can cause a larger asymmetry in the final Stokes emission at the end of the medium.

An example is shown in Fig. 5 generated by solving Eq. (8). Here the two pump pulses shown in Figs. 5(a) and 5(b) have nonsymmetric distributions of spectral amplitudes and were created using a sum of 20 frequency-shifted Gaussians of temporal width $\sigma=1 \mathrm{ps}$ with a linear ramp in amplitude spanning the bandwidth shown. All frequencies across the 

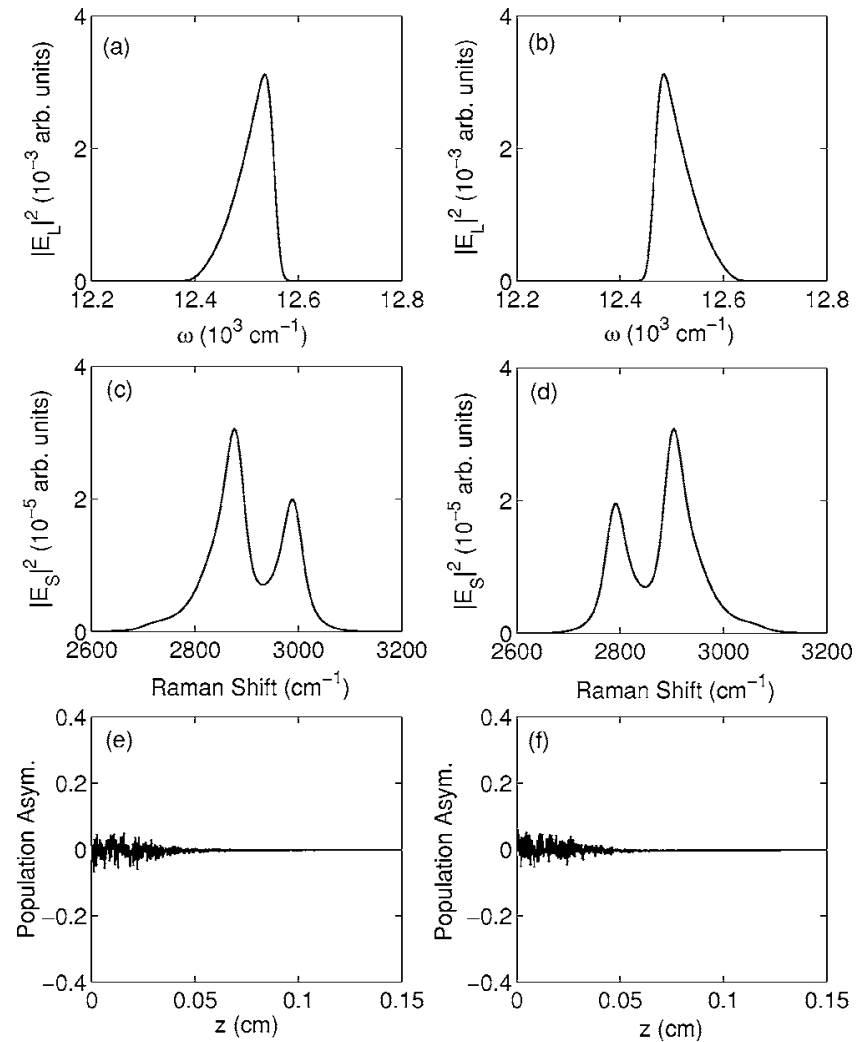

FIG. 5. Control of the Stokes emission attained by adding asymmetry to the pump spectral amplitude. (a) shows the initial pump spectrum used to generate the Stokes emission and molecular excitations shown in the left column while the pump in (b) was used to generate the Stokes emission and molecular excitations shown in the right column. (c) and (d) show the Stokes emission at the output of the medium $(z=0.15 \mathrm{~cm})$. (e) and (f) plot the final population asymmetry $\mathcal{R}\left(z, t_{f}\right)$, evaluated at the end of the pulse $t=t_{f}$, as a function of propagation length $z$.

initial bandwidth are given equal phase and the peak intensity was again set to $I_{0}=10^{11} \mathrm{~W} / \mathrm{cm}^{2}$. The remaining panels in Fig. 5 present numerical examples of control using these asymmetric pulses. For reasons elaborated below, in these examples we set the two-photon couplings equal $\left(\kappa_{21}=\kappa_{31}\right.$ $=2.8)$, neglect the collisional effects $\left(\Gamma_{2}=\Gamma_{3}=0\right)$, and include fluctuations due to spontaneous emission to seed the Raman process.

The calculated Stokes spectra, shown in Figs. 5(c) and $5(d)$, clearly show that extensive control over the ratio of the emitted Stokes lines is possible using asymmetric pump pulses. Further, although the asymmetric pump creates asymmetry in the peaks heights of the Stokes emission, there is no asymmetry in the molecular populations [Figs. 5(e) and 5(f)]. [An analytical proof demonstrating that no asymmetry in the molecular excitations is possible in the case of equal Raman mode parameters $\left(\kappa_{21}=\kappa_{31}, \Gamma_{2}=\Gamma_{3}\right.$, and $\left.\gamma_{2}=\gamma_{3}\right)$, regardless of the shape of the pump pulse, is given in the Appendix. ]

The calculated Stokes lines in Figs. 5(c) and 5(d) are seen to be shifted from their usual positions of 2837 and $2947 \mathrm{~cm}^{-1}$. These shifts are due to the fact that, since the pump pulse is asymmetrically skewed, the peak of the pump pulse no longer coincides with its mean frequency $\bar{\omega}$ $=\int \omega^{\prime}\left|E_{L}\left(z=0, \omega^{\prime}\right)\right|^{2} d \omega^{\prime}$ from which the Raman shifts are calculated. Similar shifts of the Stokes lines were seen in the optimal solutions of the adaptive feedback experiments [1-3]. However, no mechanism explaining these shifts was offered. Clearly, this is one possibility.

Note that the control mechanism demonstrated in the previous section requires that the molecular parameters $\kappa_{i 1}$ and $\Gamma_{i}$ differ for the two modes. However, the mechanism presented in this section is based on overlapping polarizations arising from the two modes, and does not require differing parameters $\kappa_{i 1}$ and $\Gamma_{i}$ for the two modes. To emphasize this last point, we have considered, in this section, the case where the two-photon couplings are equal $\left(\kappa_{21}=\kappa_{31}\right)$ and have neglected collisional effects entirely $\left(\Gamma_{2}=\Gamma_{3}=0\right)$. Thus, the control mechanism described in this section is distinctly different from that in Sec. III.

\section{SUMMARY}

Two mechanisms underlying the control of Stokes emission from two closely spaced Raman active modes have been identified. These mechanisms are likely responsible for the control of Stokes emission found in liquid methanol using adaptive feedback techniques [1-5]. The first mechanism, already present in the theory of stimulated Raman scattering in the transient regime from a single vibrational mode, relies on the fact that the uncoupled Raman gains scale differently as a function of pump pulse duration and intensity for each of the two liquid-methanol Raman modes. This control mechanism becomes active once the pulse duration of the pump becomes comparable to the collisional dephasing time scale of the Raman transitions. The second mechanism, which arises once the bandwidth of the pump pulse approaches and exceeds the energy spacing of the two Raman modes, is related to overlapping nonlinear polarizations that drive the stimulated Raman scattering. Both these mechanisms have a clear physical interpretation: shaping the pump pulse controls the nonlinear optical response of the medium, which in turn controls the stimulated Stokes emission.

It was also found, contrary to the expectations in the literature [1-5,9-13], that the vibrational populations do not reflect the ratio of peak heights seen in the Stokes spectrum. However, it is clear from Figs. 3 and 4 that a large degree of control over the population asymmetry is also possible within the present theory. Specifically, unambiguous control of the population asymmetry could be achieved experimentally if the adaptive feedback algorithm directly monitored the populations, as opposed to the current algorithm that optimizes the ratio of peak height in the scattered Stokes spectrum. For example, one could perhaps use the anti-Stokes scattering intensity of a time-delayed probe pulse $[26,27]$ as the optimization signal, a quantity which would be directly proportional to the excited-vibrational-state populations.

In the language of nonlinear optics, stimulated Raman scattering is a $\chi^{(3)}$ process. Although not discussed in this paper, we also found that additional $\chi^{(3)}$ effects, such as selfphase modulation and self-focusing, can also affect the ratio of peak heights in the Stokes spectrum. These nonlinear optics effects change the pump pulse as it propagates through 
the medium and can affect the symmetry of the pump bandwidth. The modified pump pulse bandwidth can then affect the Stokes emission through the nonlinear polarization Eq. (24) responsible for the Stokes emission. Our second study in the following paper [15] examines these ideas within the framework of a specific control experiment [4].

In addition to shedding considerable light on the experimental results, our analysis also provides insight into the nature of coherence and interference in the control, specifically the following. (1) The control can be modeled classically. Although the coupled Raman equations [Eqs. (8)] were derived using a quantum model for the vibrational modes, only the lowest-order interactions are needed to describe the stimulated Raman scattering. In this case, the same equations could have been derived using a completely classical model of the molecular vibrations $[16,20]$, where the vibrational modes are treated as classical damped harmonic oscillators. No quantum interference at the molecular level is necessary for control. (2) The control does require a pump pulse with a large and partially coherent bandwidth to achieve the control. For the first mechanism, the duration of the pump pulse must be on the order of the collisional dephasing time scales. It is then important for the pump pulse to have an envelope that is short in time, but no coherence is required of the oscillations of the electric field underneath the envelope. For the second mechanism, the pump pulse must have a large enough bandwidth to cause overlapping nonlinear polarizations arising from the two modes. Since the Stokes pulse builds up from incoherent fluctuations of the system (spontaneous decay and/or collisional initiation) leading to uncontrollable phases across the Stokes spectrum, only the amplitude profile across the pump pulse bandwidth survives as a controllable parameter. Only partial coherence is again required of the pump pulse to ensure a short-time, and hence high-intensity, temporal structure needed to drive the nonlinear process of stimulated Raman scattering. The full coherence of the pump pulse does become important when additional nonlinear propagation effects are included, as will be explored in the following paper [15].

\section{ACKNOWLEDGMENTS}

M.S. would like to acknowledge financial support from the National Science and Engineering Research Council of Canada (NSERC). This work was supported by NSERC and by Photonics Research Ontario.

\section{APPENDIX: LACK OF CONTROL OVER VIBRATIONS WITH EQUAL RAMAN MODE PARAMETERS}

In the case of $\kappa_{21}=\kappa_{31} \equiv \zeta, \Gamma_{2}=\Gamma_{3} \equiv \Gamma$, and $\gamma_{2}=\gamma_{3} \equiv \gamma$, the lack of control over the populations can be deduced analytically. Following Ref. [8], a partial solution to the coupled propagation equations [Eqs. (8)] can be constructed with the help of Laplace transforms. For simplicity, we set $\Gamma=\gamma=0$ and assume that the pump pulse changes negligibly $\left[E_{L}(z, t) \approx E_{L}(0, t) \equiv E_{L}(t)\right]$ as it propagates through the medium. The coupled equations describing the Stokes emission then reduce to

$$
\frac{\partial}{\partial z} E_{S}(z, t)=i \eta_{S} \zeta E_{L}(t)\left[Q_{21}^{*}(z, t) e^{i(\Delta / 2) t}+Q_{31}^{*}(z, t) e^{-i(\Delta / 2) t}\right],
$$

$$
\begin{gathered}
\frac{\partial}{\partial t} Q_{21}(z, t)=i \zeta E_{L}(t) E_{S}^{*}(z, t) e^{i(\Delta / 2) t}, \\
\frac{\partial}{\partial t} Q_{31}(z, t)=i \zeta E_{L}(t) E_{S}^{*}(z, t) e^{-i(\Delta / 2) t}, \\
\frac{\partial}{\partial t} Q_{22}(z, t)=-i \zeta E_{L}^{*}(t) E_{S}(z, t) e^{-i(\Delta / 2) t} Q_{21}(z, t)+\text { c . c . },
\end{gathered}
$$

$$
\frac{\partial}{\partial t} Q_{33}(z, t)=-i \zeta E_{L}^{*}(t) E_{S}(z, t) e^{i(\Delta / 2) t} Q_{31}(z, t)+\text { c . c. }
$$

Define now the Laplace transform of the field $E_{S}(z, t)$

$$
e_{S}(s, t)=\mathcal{L}\left\{E_{S}(z, t)\right\}=\int_{0}^{\infty} e^{-s z} E_{S}(z, t) d z,
$$

and similarly the transforms of the density matrix elements

$$
\begin{aligned}
& q_{21}(s, t)=\mathcal{L}\left\{Q_{21}(z, t)\right\}, \\
& q_{31}(s, t)=\mathcal{L}\left\{Q_{31}(z, t)\right\}, \\
& q_{22}(s, t)=\mathcal{L}\left\{Q_{22}(z, t)\right\}, \\
& q_{33}(s, t)=\mathcal{L}\left\{Q_{33}(z, t)\right\} .
\end{aligned}
$$

Equation (A1a) can now be solved to give

$$
e_{S}(s, t)=i s^{-1} \eta_{S} \zeta E_{L}(t)\left[q_{21}^{*}(s, t) e^{i(\Delta / 2) t}+q_{31}^{*}(s, t) e^{-i(\Delta / 2) t}\right]
$$

where the initial condition $E_{S}(z=0, t)=0$ is used. This expression for $e_{S}(s, t)$ is then substituted into the Laplacetransformed versions of Eqs. (A1b)-(A1e),

$$
\frac{\partial}{\partial t} q_{21}(s, t)=s^{-1} \zeta^{2} \eta_{S}\left|E_{L}(t)\right|^{2}\left[q_{21}(s, t)+q_{31}(s, t) e^{i \Delta t}\right],
$$

$$
\frac{\partial}{\partial t} q_{31}(s, t)=s^{-1} \zeta^{2} \eta_{S}\left|E_{L}(t)\right|^{2}\left[q_{31}(s, t)+q_{21}(s, t) e^{-i \Delta t}\right],
$$

$$
\begin{gathered}
\frac{\partial}{\partial t} q_{22}(s, t)=s^{-1} \zeta^{2} \eta_{S}\left|E_{L}(t)\right|^{2}\left[q_{21}^{*}(s, t) \otimes q_{21}(s, t)+q_{31}^{*}(s, t)\right. \\
\left.\otimes q_{21}(s, t) e^{-i \Delta t}\right]+\mathrm{c} . \mathrm{c} .,
\end{gathered}
$$




$$
\begin{aligned}
\frac{\partial}{\partial t} q_{33}(s, t)= & s^{-1} \zeta^{2} \eta_{S}\left|E_{L}(t)\right|^{2}\left[q_{31}^{*}(s, t) \otimes q_{31}(s, t)+q_{21}^{*}(s, t)\right. \\
& \left.\otimes q_{31}(s, t) e^{i \Delta t}\right]+\mathrm{c} . \mathrm{c} .,
\end{aligned}
$$

where $\otimes$ indicates a convolution over the Laplace coordinate $s$. In principle, Eqs. (A4) could be formally integrated and the inverse Laplace transform of the resulting solution could be found to give the full solution in the $z$ domain, as was done in the case of a single Raman-active mode [8]. However, the present form is adequate to display the invariance of the vibrational populations with varying the form of the pump field. The argument proceeds as follows.

First, take the complex conjugate of Eqs. (A4),

$$
\frac{\partial}{\partial t} q_{21}^{*}(s, t)=s^{-1} \zeta^{2} \eta_{S}\left|E_{L}(t)\right|^{2}\left[q_{21}^{*}(s, t)+q_{31}^{*}(s, t) e^{-i \Delta t}\right],
$$

$$
\frac{\partial}{\partial t} q_{31}^{*}(s, t)=s^{-1} \zeta^{2} \eta_{S}\left|E_{L}(t)\right|^{2}\left[q_{31}^{*}(s, t)+q_{21}^{*}(s, t) e^{i \Delta t}\right],
$$

$$
\begin{aligned}
\frac{\partial}{\partial t} q_{22}(s, t)= & s^{-1} \zeta^{2} \eta_{S}\left|E_{L}(t)\right|^{2}\left[q_{21}(s, t) \otimes q_{21}^{*}(s, t)+q_{31}(s, t)\right. \\
& \left.\otimes q_{21}^{*}(s, t) e^{i \Delta t}\right]+\mathrm{c} . \mathrm{c} .,
\end{aligned}
$$

$$
\begin{aligned}
\frac{\partial}{\partial t} q_{33}(s, t)= & s^{-1} \zeta^{2} \eta_{S}\left|E_{L}(t)\right|^{2}\left[q_{31}(s, t) \otimes q_{31}^{*}(s, t)+q_{21}(s, t)\right. \\
& \left.\otimes q_{31}^{*}(s, t) e^{-i \Delta t}\right]+ \text { c.c. }
\end{aligned}
$$

Relabeling the matrix density matrix elements as

$$
\begin{aligned}
& \alpha_{21}(s, t)=q_{31}^{*}(s, t), \\
& \alpha_{31}(s, t)=q_{21}^{*}(s, t), \\
& \alpha_{22}(s, t)=q_{33}(s, t), \\
& \alpha_{33}(s, t)=q_{22}(s, t)
\end{aligned}
$$

gives

$$
\begin{gathered}
\frac{\partial}{\partial t} \alpha_{31}(s, t)=s^{-1} \zeta^{2} \eta_{S}\left|E_{L}(t)\right|^{2}\left[\alpha_{31}(s, t)+\alpha_{21}(s, t) e^{-i \Delta t}\right], \\
\frac{\partial}{\partial t} \alpha_{21}(s, t)=s^{-1} \zeta^{2} \eta_{S}\left|E_{L}(t)\right|^{2}\left[\alpha_{21}(s, t)+\alpha_{31}(s, t) e^{i \Delta t}\right], \\
\frac{\partial}{\partial t} \alpha_{33}(s, t)=s^{-1} \zeta^{2} \eta_{S}\left|E_{L}(t)\right|^{2}\left[\alpha_{31}^{*}(s, t) \otimes \alpha_{31}(s, t)+\alpha_{21}^{*}(s, t)\right. \\
\left.\otimes \alpha_{31}(s, t) e^{i \Delta t}\right]+\mathrm{c} . \mathrm{c} .,
\end{gathered}
$$

$$
\begin{aligned}
\frac{\partial}{\partial t} \alpha_{22}(s, t)= & s^{-1} \zeta^{2} \eta_{S}\left|E_{L}(t)\right|^{2}\left[\alpha_{21}^{*}(s, t) \otimes \alpha_{21}(s, t)+\alpha_{31}^{*}(s, t)\right. \\
& \left.\otimes \alpha_{21}(s, t) e^{-i \Delta t}\right]+\mathrm{c} \mathrm{c} \mathrm{c} .
\end{aligned}
$$

Upon comparing Eqs. (A7) with Eqs. (A4), it can been seen that, given a first solution

$$
\mathcal{S}^{(1)}=\left\{q_{21}^{(1)}(s, t), q_{31}^{(1)}(s, t), q_{22}^{(1)}(s, t), q_{33}^{(1)}(s, t)\right\},
$$

a second solution of the form

$$
\begin{aligned}
\mathcal{S}^{(2)} & =\left\{q_{21}^{(2)}(s, t), q_{31}^{(2)}(s, t), q_{22}^{(2)}(s, t), q_{33}^{(2)}(s, t)\right\} \\
& =\left\{q_{31}^{(1) *}(s, t), q_{21}^{(1) *}(s, t), q_{33}^{(1)}(s, t), q_{22}^{(1)}(s, t)\right\}
\end{aligned}
$$

exists. Given the initial conditions at $t=0$ that lead to the first solution, the second solution is then constructed by switching the indices $2 \leftrightarrow 3$ and taking the complex conjugate. In the position domain, instead of the Laplace domain, these two sets of initial conditions are connect by initial conditions

$$
\begin{aligned}
& Q_{21}^{(2)}(z, t=0)=\left[Q_{31}^{(1)}(z, t=0)\right]^{*}, \\
& Q_{31}^{(2)}(z, t=0)=\left[Q_{21}^{(1)}(z, t=0)\right]^{*}, \\
& Q_{22}^{(2)}(z, t=0)=\left[Q_{33}^{(1)}(z, t=0)\right], \\
& Q_{33}^{(2)}(z, t=0)=\left[Q_{22}^{(1)}(z, t=0)\right],
\end{aligned}
$$

which then leads to the two solutions connected by

$$
\begin{aligned}
& Q_{21}^{(2)}(z, t)=\left[Q_{31}^{(1)}(z, t)\right]^{*}, \\
& Q_{31}^{(2)}(z, t)=\left[Q_{21}^{(1)}(z, t)\right]^{*}, \\
& Q_{22}^{(2)}(z, t)=\left[Q_{33}^{(1)}(z, t)\right], \\
& Q_{33}^{(2)}(z, t)=\left[Q_{22}^{(1)}(z, t)\right] .
\end{aligned}
$$

The existence of these two connected solutions has been verified numerically by applying the transformation of Eqs. (A8) to a given set of initial conditions.

The buildup of energy in the Stokes mode starts from, for example, spontaneous emission. In the present model, spontaneous emission is included through stochastic initial conditions imposed on $Q_{21}(z, t=0)$ and $Q_{31}(z, t=0)$. These quantities are each initialized to small random complex numbers with a Gaussian distribution in amplitude. For each solution found using a particular set of stochastic initial conditions leading to a particular final population asymmetry

$$
\mathcal{R}^{(1)}\left(z, t_{f}\right)=\frac{Q_{22}^{(1)}\left(z, t_{f}\right)-Q_{33}^{(1)}\left(z, t_{f}\right)}{Q_{22}^{(1)}\left(z, t_{f}\right)+Q_{33}^{(1)}\left(z, t_{f}\right)},
$$

the connected second solution from Eqs. (A9) will lead to the exact opposite population asymmetry,

$$
\begin{aligned}
\mathcal{R}^{(2)}\left(z, t_{f}\right) & =\frac{Q_{22}^{(2)}\left(z, t_{f}\right)-Q_{33}^{(2)}\left(z, t_{f}\right)}{Q_{22}^{(2)}\left(z, t_{f}\right)+Q_{33}^{(2)}\left(z, t_{f}\right)}=\frac{Q_{33}^{(1)}\left(z, t_{f}\right)-Q_{22}^{(1)}\left(z, t_{f}\right)}{Q_{33}^{(1)}\left(z, t_{f}\right)+Q_{33}^{(1)}\left(z, t_{f}\right)} \\
& =-\mathcal{R}^{(1)}\left(z, t_{f}\right) .
\end{aligned}
$$

Furthermore, the two solutions connected by the initial con- 
ditions Eq. (A8) both appear with equal weights in the initial distribution. This implies that every point in the initial distribution has a related point with exactly the same weight and exactly the opposite population asymmetry. Hence, after averaging over all the initial conditions needed to model the spontaneous emission, the final population asymmetry of the vibrations must be zero- there is no possibility for control over the relative populations of the vibrational states. This constraint on the relative populations, however, still leaves open room for controlling the temporal shape (i.e., bandwidth) of the Stokes emission, even though the vibrational energy (i.e., population) is, on average, distributed equally between the two modes. A similar set of arguments showing that the populations must be equal holds if the Raman modes are seeded with the collisional fluctuations instead of spontaneous emission.
[1] T. C. Weinacht, J. L. White, and P. H. Bucksbaum, J. Phys. Chem. A 103, 10166 (1999).

[2] B. J. Pearson, J. L. White, T. C. Weinacht, and P. H. Bucksbaum, Phys. Rev. A 63, 063412 (2001).

[3] T. C. Weinacht and P. H. Bucksbaum, J. Opt. B: Quantum Semiclassical Opt. 4, R35 (2002).

[4] B. J. Pearson and P. H. Bucksbaum, Phys. Rev. Lett. 92, 243003 (2004).

[5] J. L. White, B. J. Pearson, and P. H. Bucksbaum, J. Phys. B 37, L399 (2004)

[6] R. L. Carman, M. E. Mack, F. Shimizu, and N. Bloembergen, Phys. Rev. Lett. 23, 1327 (1969).

[7] M. J. Colles and J. E. Griffiths, J. Chem. Phys. 56, 3384 (1972).

[8] M. G. Raymer and J. Mostowski, Phys. Rev. A 24, 1980 (1981).

[9] H. Rabitz and W. Zhu, Acc. Chem. Res. 33, 572 (2000).

[10] J. M. Geremia and H. Rabitz, Phys. Rev. Lett. 89, 263902 (2002).

[11] I. Walmsley and H. Rabitz, Phys. Today 56 (8), 43 (2003).

[12] M. Dantus and V. V. Lozovoy, Chem. Rev. (Washington, D.C.) 104, 1813 (2004).

[13] T. Brixner, G. Krampert, T. Pfeifer, R. Selle, G. Gerber, M. Wollenhaupt, O. Graefe, C. Horn, D. Liese, and T. Baumert, Phys. Rev. Lett. 92, 208301 (2004).

[14] B. J. Pearson and P. H. Bucksbaum, Phys. Rev. Lett. 94, 209901(E) (2005).
[15] M. Spanner and P. Brumer, following paper, Phys. Rev. A 73, 023810 (2006).

[16] C. S. Wang, Phys. Rev. 182, 482 (1969).

[17] R. L. Carman, F. Shimizu, C. S. Wang, and N. Bloembergen, Phys. Rev. A 2, 60 (1970).

[18] C. S. Wang, in Quantum Electronics, edited by H. Rabin and C. L. Tang (Academic Press, New York, 1975), Vol. 1, Pt. A, p. 447.

[19] E. E. Hagenlocker, R. W. Mink, and W. G. Rado, Phys. Rev. 154, 226 (1967).

[20] R. W. Boyd, Nonlinear Optics (Academic Press, San Diego, 1992).

[21] T. C. Weinacht, R. Bartels, S. Backus, P. H. Bucksbaum, B. Pearson, J. M. Geremia, H. Rabitz, H. C. Kapteyn, and M. M. Murnane, Chem. Phys. Lett. 344, 333 (2001).

[22] R. A. Bartels, T. C. Weinacht, S. R. Leone, H. C. Kapteyn, and M. M. Murnane, Phys. Rev. Lett. 88, 033001 (2002).

[23] R. A. Bartels, S. Backus, M. M. Murnane, and H. C. Kapteyn, Chem. Phys. Lett. 374, 326 (2003).

[24] M. Lewenstein, Z. Phys. B: Condens. Matter 56, 69 (1984).

[25] CRC Handbook of Chemistry and Physics, edited by D. R. Lide (CRC Press, Boca Raton, FL, 2002).

[26] A. Laubereau, D. von der Linde, and W. Kaiser, Phys. Rev. Lett. 28, 1162 (1972).

[27] L. K. Iwaki and D. D. Dlott, Chem. Phys. Lett. 321, 419 (2000). 